\title{
Les centres de qualification d'équipements de sécurité nucléaire du CEA: \\ leur apport à la sûreté des installations nucléaires et à la protection des travailleurs *
}

\author{
P. LEBOULEUX, R. PRIGENT, C. ROUSSEL** \\ (Manuscrit reçu le 28 juin 1988)
}

\begin{abstract}
RÉSUMÉ
L'Institut de protection et de sûreté nucléaire du Commissariat à l'énergie atomique (CEA) s'est doté de centres de qualification pour les équipements liés à la sûreté des installations et à la protection des travailleurs. Ces centres créés pour coordonner les moyens du CEA et de ses filiales notamment dans le domaine des essais ont pour missions principales l'homologation d'équipements, de moyens de calcul et de procédés; la participation à la normalisation nationale et internationale; les actions de formation spécialisée; la centralisation et la diffusion de l'information disponible.

A ce jour, trois centres ont été mis sur pieds: le Centre technique d'homologation de l'instrumentation de radioprotection (CTHIR), le Centre technique de référence en ventilation et épuration des effluents gazeux (CETREVE), et le Centre technique d'homologation des équipements de protection, de manipulation, de détection et de sécurité (CTHPMDS).

Ils couvrent les domaines suivants: confinement, ventilation, épuration de l'air ou des effluents gazeux; instrumentation de radioprotection et de sécurité des installations et de l'environnement; dispositifs et vêtements de protection, blindages, dispositifs de transfert de matériaux radioactifs, téléopération, télémanipulation et transferts. Ces centres jouent un rôle important pour la protection et la sûreté nucléaire en agissant sur la qualité des équipements et matériels, moyens de calcul et procédés en vérifiant notamment l'adéquation des produits aux besoins exprimés, sur la qualité de l'exploitation en participant à la formation du personnel et en assurant la prise en compte du retour d'expérience, sur la qualité de l'intervention dans les situations accidentelles et postaccidentelles.
\end{abstract}

* Communication présentée lors de la Conférence internationale sur la radioprotection et l'énergie nucléaire, organisée par l'AIEA, à Sydney, Australie, 18-22 avril 1988.

** Commissariat à l'énergie atomique, Institut de protection et de sûreté nucléaire, Département de protection technique, BP 6, 92265 Fontenay-aux-Roses Cedex (France). 


\title{
ABSTRACT
}

\begin{abstract}
The Institute for radiation protection and nuclear safety (IPSN) of the Commissariat à l'énergie atomique (CEA) has set up testing centres for equipment connected with the safety of facilities and staff protection. These centres, established in order to co-ordinate the resources of the CEA and of its subsidiaries, particularly in relation to testing, are responsible, each within its own field of activity, for the following main tasks: approval of equipment, calculation techniques and processes; participation in national and international standardization work; specialized training activities; centralization and dissemination of available information. So far, three centres have been set up and are playing an important role in radiation protection and nuclear safety through their work in relation to: the quality of equipment and materials, calculation techniques and processes, ensuring in particular that products are adequate to meet the needs expressed; operational quality, through participation in staff training and by ensuring that the feedback of experience is taken into account; the quality of the actions undertaken in accident and post-accident situations.
\end{abstract}

\section{LA QUALIFICATION DES OUTILS DE LA SÉCURITÉ NUCLÉAIRE AU CEA}

Qualifier par des essais de différents types les matériels, codes de calcul et procédés n'est pas une pratique nouvelle; depuis longtemps des installations d'essais de toute nature effectuent à la demande de concepteurs réalisateurs ou à la demande d'utilisateurs les essais en question.

Le Commissariat à l'énergie atomique (CEA) a développé pour les besoins de la recherche nucléaire un grand nombre d'installations qui lui ont permis de valider des procédés et des codes de calculs. Les résultats de ces travaux sont mis en œuvre par l'industrie nucléaire et l'utilisateur - l'exploitant nucléaire, au sens de la réglementation française - est soumis aux règles très strictes imposées par les pouvoirs publics pour la délivrance des autorisations réglementaires.

Le CEA, à la fois metteur au point de matériels, codes ou procédés et exploitant d'installations nucléaires d'essai ou de production, a jugé nécessaire de se doter des outils nécessaires pour coordonner les moyens déjà en place et répondre ainsi, sans engager de dépenses importantes, aux exigences de plus en plus précises de qualité et de démonstration de l'obtention de la qualité. Compte tenu de l'objectif fixé, l'Institut de protection et de sûreté nucléaire (IPSN) s'est chargé de l'opération. La création de centres techniques de qualification s'est effectuée au fur et à mesure des besoins et des possibilités. Les missions principales de ces centres explicitées plus loin recouvrent les objectifs suivants:

- homologation d'équipements, de moyens de calcul et de procédés,

- participation à la normalisation nationale et internationale,

- actions de formation spécialisée,

- centralisation et diffusion de l'information disponible.

Un aspect fondamental du mode d'action de ces centres est qu'ils agissent pour le compte du groupe CEA (le CEA et ses filiales) et avec les moyens du groupe CEA. D'autre part ils ne visent à qualifier que les 
caractéristiques des équipements, codes ou procédés en rapport avec la sécurité nucléaire. Les moyens propres de chacun de ces centres de qualification sont très réduits: deux ingénieurs et un secrétariat à temps partiel, mais ils s'appuient sur les laboratoires du groupe CEA et sur les experts des unités qui font appel à leur service. La démarche des unités du groupe CEA est d'ailleurs purement volontaire et c'est par leur efficacité que les centres de qualification se sont imposés.

\section{LES CENTRES TECHNIQUES DE QUALIFICATION}

Trois centres de qualification sont actuellement opérationnels :

\subsection{Le Centre technique d'homologation de l'instrumentation de radioprotection (CTHIR) (fig. 1).}

Créé en 1981 après une période de réflexion d'environ deux ans, son domaine d'activité est l'instrumentation de radioprotection: appareils de détection, de mesure et d'alarme à poste fixe, mobiles ou portés par le personnel dans les installations nucleaires, appareils de surveillance de la radioactivité dans l'environnement, équipements de centralisation des informations recueillies. Pour apprécier l'aptitude des instruments de radioprotection à assurer leur fonction, le CTHIR:

- définit les essais permettant de vérifier les caractéristiques et les performances des appareils en s'appuyant sur les textes normatifs et les spécifications particulieres définies pour ces appareils;

- fait exécuter les essais conformément à des modes opératoires agréés;

- analyse les résultats obtenus et délivre le cas échéant un certificat d'homologation;

- fait prendre les mesures nécessaires pour assurer la conformité des appareils de série aux prototypes soumis à ses investigations.

En ce qui concerne l'organisation générale de son action, le CTHIR est en liaison notamment avec:

- un comité d'instrumentation de la radioprotection représentant les utilisateurs, pour définir les besoins du groupe CEA;

- un comité consultatif en matière de politique industrielle de l'instrumentation de radioprotection, qui définit la politique industrielle du groupe CEA en la matière;

- des laboratoires d'essais du groupe CEA agréés par lui et actuellement en cours d'accréditation par le Réseau national d'essais (1);

- les industriels fabriquant des matériels.

(1) Le Réseau national d'essais gère un système d'accréditations de laboratoires d'essais basées sur la valeur et la qualité des prestations d'essais de ces laboratoires. 

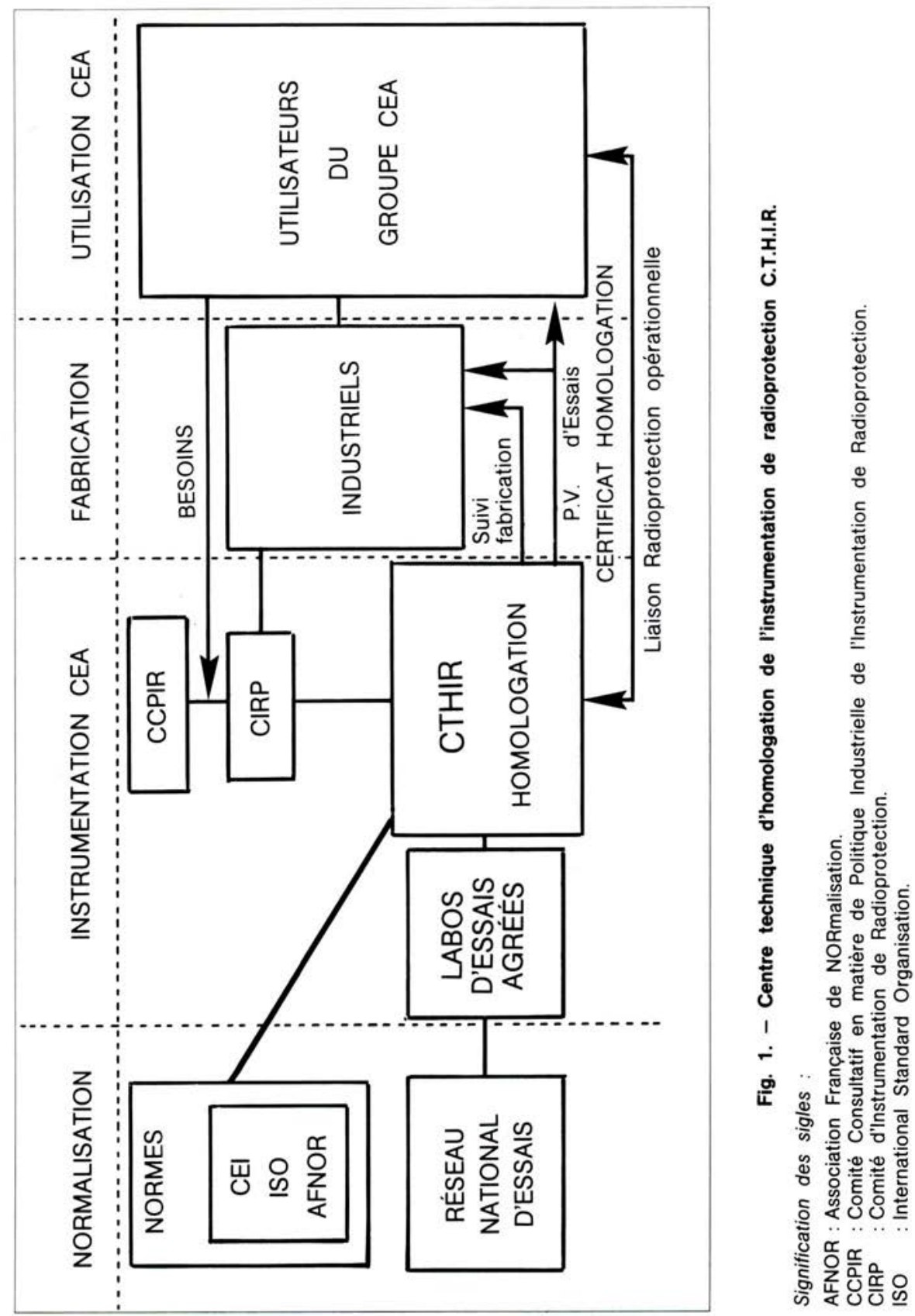
Une procédure précise le rôle des divers intervenants et les conditions dans lesquelles une homologation peut être délivrée.

Compte tenu de son expérience en la matière, le CTHIR participe largement aux travaux de normalisation nationaux et internationaux, en particulier ceux effectués dans le cadre du sous-comité $45 \mathrm{~B}$ de la Commission électrotechnique internationale (instrumentation pour la radioprotection).

\subsection{Le Centre technique de référence en ventilation et épuration (CETREVE).}

Le domaine d'activité de ce centre, créé en 1982, est centré sur la ventilation et l'épuration et comprend notamment la conception générale de la ventilation, la filtration à très haute efficacité, le piégeage des iodes et des composés volatils, la préfiltration et filtration électrostatique et les applications spéciales, la fonction de la ventilation en cas d'incendie, la ventilation et la détection de la contamination, les enceintes de confinement, l'épuration des gaz de procédé.

Le CETREVE prononce des homologations de matériels ou de codes de calcul dans des conditions voisines de celles du CTHIR. De plus, il a développé des actions de formation spécialisée et de diffusion de l'information particulièrement importantes.

Les sessions de formation d'une semaine animées par le CETREVE, au nombre de 5 à 6 par an, s'adressent aux ingénieurs et techniciens chargés de la conception, de la réalisation, de l'exploitation ou des essais des circuits de ventilation et d'épuration. Cette formation très pragmatique vise, d'une part, à assurer la qualification du personnel en place et, d'autre part, à faciliter la reconversion de nouveaux agents. Ne réunissant qu'un petit nombre de participants à la fois, elles permettent, en outre, des échanges d'informations et constituent un excellent canal pour le retour d'expérience.

En ce qui concerne l'information, le CETREVE est la cheville ouvrière de l'élaboration d'un guide de ventilation des installations nucléaires rédigé par une équipe constituée de représentants de l'ensemble de la profession et qui recense les solutions techniques disponibles, laissant aux utilisateurs le soin de choisir celle d'entre-elles qui répondra le mieux au problème posé [2]. Le CETREVE va développer maintenant un guide de l'épuration en s'inspirant des mêmes principes.

\subsection{Le Centre technique d'homologation des équipements de protection, de manipulation, de détection et de sécurité (CTHPMDS).}

Ce centre est le dernier en date puisque créé en 1985. Son domaine d'activité est plus vaste et plus diversifié que celui des autres centres et concerne notamment le confinement, les blindages, l'instrumentation de sécurité, les télémanipulateurs et leur équipements associés, les transferts de matières radioactives. 
Le CTHPMDS procède, comme les autres centres, à des actions d'homologation dans des conditions un peu différentes compte tenu de sa spécificité. En effet, seules sont généralement disponibles des notices des constructeurs et des méthodes générales d'essais à adapter aux besoins particuliers. II y a donc lieu de rédiger d'abord des spécifications techniques du matériel à homologuer après une enquête approfondie sur les besoins, puis de définir des méthodes d'essai et des critères de jugement pour vérifier l'aptitude du matériel à satisfaire les besoins exprimés. Pour ce faire, le CTHPMDS s'appuie sur :

- une commission centrale regroupant des représentants des centres d'études nucléaires et des établissements du groupe CEA qui définit un programme d'homologation; ques;

- des groupes de travail sectoriels qui rédigent les spécifications techni-

$$
\begin{aligned}
& \text { - des laboratoires d'essais du groupe CEA, reconnus par le centre } \\
& \text { technique. }
\end{aligned}
$$

Le CTHPMDS agit, par ailleurs, en liaison permanente avec les constructeurs concernés. Notons, en outre, qu'il est l'aboutissement d'une action de normalisation et de standardisation des équipements de protection et de sécurité au CEA menée depuis une vingtaine d'années et gérée par la Commission centrale déjà citée. La nécessité d'une formalisation des actions de qualification a été plus vivement ressentie avec la sortie, en 1984, de la réglementation française en matière de qualité des installations nucléaires [3].

\section{APPORT DES CENTRES DE QUALIFICATION Ȧ LA SÛRETÉ NUCLÉAIRE ET À LA RADIOPROTECTION DES INSTALLATIONS}

\subsection{Qualité des équipements, moyens de calcul et procédés}

La pratique française en matière de qualité des installations nucléaires repose sur la mise en œuvre du code de bonne pratique de l'AIEA [1]. Cette pratique a été confirmée par le texte réglementaire français cité [3]. Ce règlement précise que l'exploitant d'une installation nucléaire doit montrer que la qualité définie pour les équipements importants pour la sûreté a été obtenue et qu'elle est maintenue.

Le certificat d'homologation, délivré après des essais indépendants dont la qualité est elle-même attestée, est un élément essentiel de cette démonstration. Toutefois, l'expérience a montré que, même sur de très bons équipements, des actions correctives étaient nécessaires sur des points particuliers et que l'étude du fonctionnement en situation réelle constituait un complément indispensable aux essais de qualification. Pour intégrer et assurer l'efficacité de ces actions, le certificat d'homologation n'est accordé qu'un an après les essais de qualification proprement dits.

II n'est pas dans l'intention des centres de qualification d'homologuer tout le matériel de sécurité utilisé au CEA mais, en priorité, les nouveaux 
matériels développés et, ensuite, les matériels déjà en usage mais pour lesquels il y a des doutes sur le maintien à niveau d'une fourniture, ou en raison de la prise en compte de nouveaux critères.

\subsection{Qualité de l'exploitation}

La qualité de l'exploitation d'une installation repose sur un grand nombre de facteurs: parmi eux, l'adéquation aux besoins des équipements liés à l'exploitation, la formation du personnel et le retour d'expérience.

La formation du personnel nécessite plusieurs composantes. Les centres de qualification n'interviennent en la matière que dans leur domaine propre c'est-à-dire principalement l'utilisation et la maintenance des équipements de sécurité. A l'exception des sessions da'CETREVE gérées par le centre luimême avec l'appui de formateurs issus de toute la profession, ces actions sont très ponctuelles. Le principe est de former de préférence des formateurs. Par exemple, avec la Compagnie générale des matières nucléaires (Cogéma), un système de formation a été mis au point au profit des opérateurs manipulant les dispositifs de transfert blindés de déchets extraits des cellules de haute activité. L'enseignement est entièrement assuré par des agents de la Cogéma, mais le centre de qualification a participé à la formation des moniteurs, à la définition du programme de formation et a fourni la documentation. Des rendez-vous périodiques permettent un suivi et le retour d'expérience.

Ce dernier point est aussi un souci constant des centres de qualification qui développent des relations avec les constructeurs, avec les utilisateurs et favorisent les contacts entre constructeurs et utilisateurs, et entre utilisateurs. Les résultats de cette concertation se traduisent dans les publications des centres dont nous avons donné des exemples ainsi que par le soutien à l'effort normatif national et international.

\subsection{Qualité de l'intervention en situation accidentelle et post-accidentelle}

Le CEA, et notamment I'IPSN, est un appui technique des pouvoirs publics en cas d'accident, entre autres par les moyens d'intervention dont il dispose. Ces moyens doivent être opérationnels sans délai, qu'ils soient déjà sur place au moment où l'accident survient (par exemple: pièges à iode, détecteurs d'irradiation ou de contamination à large gamme) ou qu'ils soient utilisés par les équipes d'intervention (instrumentation de radioprotection, moyens de téléintervention et de téléopération, dispositifs de protection individuels, etc.). Les différents centres de qualification sont concernés et l'on peut citer, à titre d'exemple, les actions suivantes:

Pour le CTHIR, homologation des équipements suivants:

- chambre d'ionisation $\gamma$ (CHFG) avec une étendue de mesure allant jusqu'à $10^{7}$ rad. $h^{-1}$;

- télédébimètre (TELETECTOR) mesurant jusqu'à $10^{3}$ rad. $^{-1}$; 
- capteurs pour la mesure de l'irradiation $\gamma$ et neutrons (CIEP et CINN) avec une gamme de mesure de $10^{-1}$ à $10^{3}$ rem. $\mathrm{h}^{-1}$;

- balise $\alpha$ à filtre séquentiel (BFSA) et $\beta$ (BFSB) pouvant mesurer de 0,1 à $10^{4} \mathrm{LDCA}$ (2) (plutonium 239 pour les $\alpha$ et strontium 90 pour les $\beta$ ).

En cours d'homologation:

- balise de chantier pour l'intervention.

\section{Pour le Cetreve:}

Les filtres et les pièges à iode font depuis longtemps l'objet d'une procédure spécifique appliquée sous la seule responsabilité des fournisseurs. Une procédure d'homologation va leur être appliquée, qui intégrera d'une part des essais plus complets (par exemple, tenue des filtres pendant un incendie) et la mise en place d'un système d'assurance de la qualité.

\section{Pour le CTHPMDS :}

Un certain nombre d'engins de téléintervention et de téléopération vont être soumis à des essais de tenue sous rayonnement pour des doses de rayonnement pouvant atteindre de $10^{6}$ à $10^{7}$ rads. Des vêtements de protection de type divers, adaptés à l'intervention, vont également être soumis à I'homologation.

\section{CONCLUSION}

Une structure légère a été mise en place au CEA pour coordonner et améliorer l'efficacité des moyens de qualification des équipements de sécurité nucléaire. Cette structure est à la disposition des exploitants du CEA et ses actions reposent principalement sur les laboratoires du CEA tout en maintenant des contacts étroits avec les industriels. Elle fonctionne sur la base du volontariat des participants, tout en mettant en œuvre des procédures et des moyens techniques qui garantissent la qualité des interventions. En facilitant la maîtrise de la qualité des équipements de sécurité nucléaire, les centres de qualification du CEA/IPSN apportent leur contribution à la sûreté nucléaire et à la radioprotection des installations, notamment pour l'exploitation et pour les interventions dans des circonstances accidentelles et postaccidentelles.

\section{RÉFÉRENCES}

[1] AGENCE INTERNATIONALE DE L'ÉNERGIE ATOMIQUE (AIEA). Assurance de la qualité pour la sûreté des centrales nucléaires. (Collection Sécurité, $n^{\circ} 50-C-Q A$ ). Vienne: AIEA, 1979.

[2] COMMISSARIAT À L'ÉNERGIE ATOMIQUE (CEA). Guide de ventilation des installations nucléaires, 2. éd. Boulogne-Billancourt: ETAI, 1987.

[3] FRANCE. Arrêté et circulaire du 10 août 1984 relatifs à la qualité de la conception, de la construction et de l'exploitation des installations nucléaires de base. J.O.N.C., 22 sept. 1984, N.222, 8652-8658.

(2) Limite dérivée de concentration d'un radionucléide dans l'air. 